\title{
On the "initial" Angular Momentum of Galaxies
}

\author{
Tom Abel, Rupert C. Croft, Lars Hernquist \\ Harvard Smithsonian Center for Astrophysics, \\ MA, US-02138 Cambridge \\ E-mail: Hi@TomAbel.com
}

\begin{abstract}
Spherical density profiles and specific angular momentum profiles of Dark Matter halos found in cosmological N-body simulations have been measured extensively. The distribution of the total angular momentum of dark matter halos is also used routinely in semi-analytic modeling of the formation of disk galaxies. However, it is unclear whether the initial (i.e. at the time the halo is assembled) angular momentum distributions of baryons is related to the dark matter at all. Theoretical models for ellipticities in weak lensing studies often rely on an assumed correlation of the angular momentum vectors of dark matter and gas in galaxies. Both of these assumptions are shown to be in reasonable agreement with high resolution cosmological smoothed particle hydrodynamical simulations that follow the dark matter as long as only adiabatic gas physics are included. However, we argue that in more realistic models of galaxy formation one expects pressure forces to play a significant role at "turn-around". Consequently the torquing force on DM and baryons will be uncorrelated and their respective angular momenta are not expected to align. An SPH simulation with ad-hoc feedback is presented that illustrates these effects. Massive low redshift elliptical galaxies may be a notable exception where "light may trace mass".
\end{abstract}

\section{Introduction}

The high frequency of disk galaxies and their exponential profiles over many scale radii is perhaps one of the most striking global features of observed galaxies. Theoretical studies investigating the origin of this angular momentum in these galaxies have a long history (e.g. Weizsäcker 1951). In most modern investigations the Ansatz of Mestel (1963) is used where one assumes that any parcel of fluid to retains its initial specific angular momentum. In hierarchical structure formation scenarios the luminous parts of galaxies form from gas that is cooling within a dark matter (DM) halos that continuously are merging to build larger and larger objects. These assumption are made also in most recent models of disk galaxies (e.g. Mo, Mao and White 1998). The typical initial angular momentum of halos may be estimated from linear theory (Peebles 1969). However, its distribution is typically measured from $\mathrm{N}$-body simulations that follow the formation and evolution of structure in the dark matter only. In the following we derive a rough estimate of the the magnitude of pressure forces that contribute significantly to the torquing forces. 


\section{Forces and Torques}

The critical density and the total mass, $M$, in a spherical volume of comoving radius $R$ with an over-density $\delta=\rho / \bar{\rho}_{m}$ of the mean matter density $\left(\bar{\rho}_{m}=\right.$ $\left.\Omega_{m} \rho_{c}\right)$ are given by,

$$
\rho_{c}=\frac{3 H_{0}^{2}}{8 \pi G}, \quad M=\delta \frac{4 \pi}{3} R^{3} \Omega_{m} \rho_{c},
$$

where the symbols have their usual meaning (see e.g. Peebles 1993). Let $f$ be the fraction of the torquing force from surrounding structures on this spherical over-density to the radial force of the sphere. I.e. the torquing force is $F_{t}=f G M \rho / r^{2}$ where $r$ is a proper radius of the sphere. Here $f$ is necessarily somewhat smaller than the radial force of its own gravity 9 . To get a rough idea of what pressure gradient may change the torquing we simply compare

$$
\frac{1}{\rho_{b}} \frac{\partial P}{\partial r}=f \frac{G M}{r^{2}}
$$

Using difference instead of differential, substituting $r=R /(1+z)$ and using $\triangle R$ F to denote the comoving length scale over which the pressure gradient is taken we find,

$$
\triangle P \sim f \frac{3 H_{0}^{4}}{16 G \pi} \delta^{2} \Omega_{b} \Omega_{m}(1+z)^{4} R \triangle R .
$$

Assuming constant density this translates to the very small temperature gradient of

$$
\begin{aligned}
& \triangle T \sim f \frac{\triangle P \mu}{k_{B} \rho_{b}}=\delta \frac{H_{0}^{2} m_{P}}{2 k_{B}} \Omega_{m}(1+z) R \triangle R, \\
& \triangle T \approx 2.6 \times 10^{3} \mathrm{~K} \delta \frac{f}{0.1}(1+z) R_{M p c} \frac{\triangle R_{M p c}}{0.3} .
\end{aligned}
$$

Such small pressure gradients are expected to originate from well known processes such as ionization fronts from reionization, galactic outflows driven by black holes, and galactic feedback as required in semi-analytical models of galaxy formation (e.g. Kauffmann et al. 2000). The larger the mass of the object the larger the pressure gradient has to be to alter the angular momentum acquisition of the gas as compared to the dark matter.

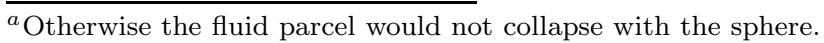

${ }^{b}$ This $\delta R$ is taken smaller than the total radius since for a realistic density distribution most of the mass is at large radii. 


\section{Simulations}

To investigate the relation between angular momentum of the baryons and the DM we have performed two different cosmological smoothed particle hydrodynamics simulations using GADGET (Springel, Yoshida, and White 2000). Both simulations use $128^{3}$ for each the DM and the gas in a periodic volume of 10 comoving Mpc side-length. Both are evolved from redshift 60 to $z=3$ with initial conditions drawn from a realization appropriate for a spatially flat universe with $\Omega_{m}=0.3, \Omega_{b} h^{2}=0.02$ and $h=0.7$. Both simulations follow only gravity and adiabatic gas physics. In one run we have artificially increased abruptly the temperature of the gas to $4 \times 10^{6}$ Kelvin at redshift 7 to mimic a strong feedback case. We use HOP (Eisenstein and Hut 1998) to identify

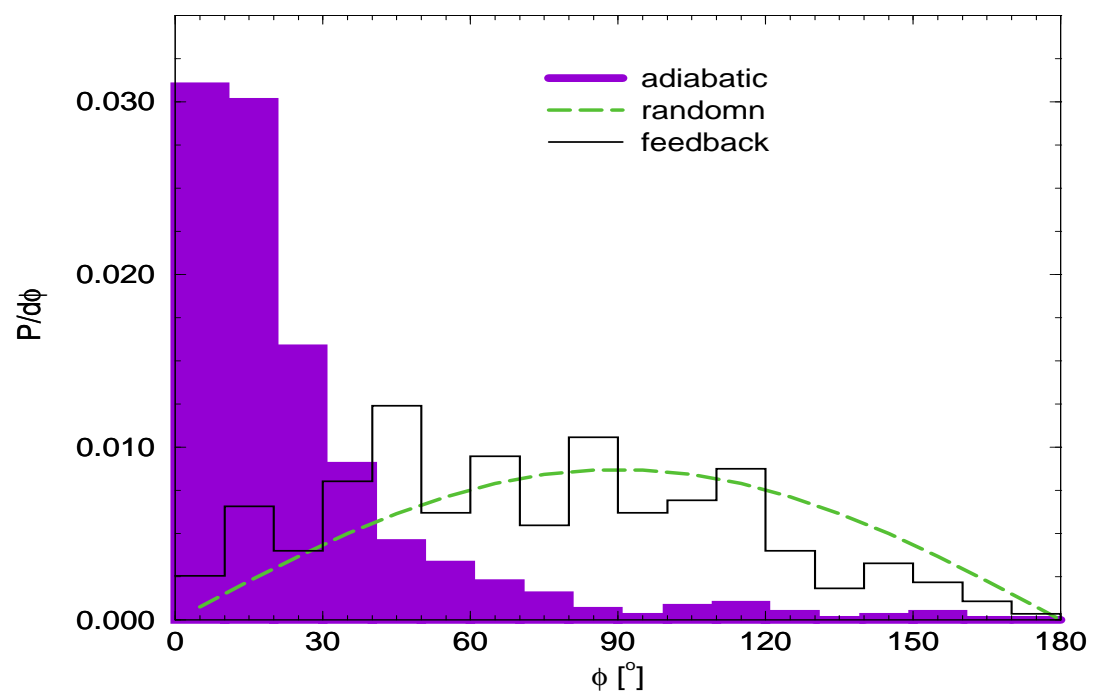

Figure 1: Alignment angle between the total specific angular momentum vectors of dark matter and gas for purely adiabatic gas physics (filled histogram) and an ad-hoc feedback model (empty histogram). The smooth dashed line indicates the expectation for a purely random distribution.

centers of halos. The we go out radially and define a halo as all the mass within the radius where the over-density in the dark matter corresponds to 200 times the mean density. Within these halos we then measure the total and specific angular momentum vectors. In Figure 1 we show the distributions of the alignment angles between the dark matter and the baryonic component for all halos that have no other overlapping halo. Clearly for the purely adiabatic case their is remarkable agreement between the directions of the angular momenta with a median alignment angle of 20 degrees. However, as soon as 
pressure forces are introduces as in the ad-hoc feedback the respective angles become uncorrelated. The imperfect alignment between the DM and the gas in the purely adiabatic run comes most likely from non-linear angular momentum exchange at virialization. Such non-linear effects may well be aggravated in simulations which also include the cooling of the gas.

\section{Discussion and Conclusions}

In this short contribution we have pointed out that large scale pressure forces have significant influence on the formation of galaxies. Until the physical processes acting on the gas in the intergalactic medium are fully understood it is difficult to asses up to which mass scale such large scale non-gravitational forces may play a role. Because of space constraints we could only remark here that the collisionless and collisional components will exchange angular momentum during merging events. This will further diminish and possible correlation of the spins of DM and gas. Our results have important implications for models which associate the dark matter angular momentum vector and their correlations with observed ellipticities of galaxies (see contributions by Natarayan, Crittenden and Heavens in this volume). Since the directions of the angular momentum of radial shells within halos varies as function of radius (Moore this volume) and the luminous part of galaxies may be re-oriented from the torquing their non-spherical parent halos (Moore and Sellwood, this volume) we are led to conclude that current models of the formation and evolution of disk galaxies are oversimplified. However, one interesting exception may be low redshift massive elliptical galaxies. If most of their stars have formed at early times in smaller higher redshift objects the shapes and angular momentum distributions of gas and DM may be strongly correlated. This would be a natural explanation of the strong lensing observations of Kochaneck (this volume) which indicate that "light traces mass" for these galaxies.

\section{References}

1. Eisenstein, D. J. \& Hut, P., ApJ 498, 137 (1998)

2. Kauffmann, G., Colberg, J. M., Diaferio, A., \& White, S. D. M., MNRAS 303, 188 (1999)

3. Mestel, L., MNRAS 126, 553 (1963)

4. Mo, H. J., Mao, S., \& White, S. D. M., MNRAS 295, 319 (1998)

5. Peebles, P. J. E., ApJ 155, 393 (1969)

6. Springel, V., Yoshida, N., White, S. D. M., astro-ph/0003162, 2000

7. von Weizsäcker, C. F. ApJ 114, 165 (1951) 